\title{
Gamma-emitting Short-lived Nuclides Formed by Neutron Activation
}

\author{
Minoru OKADA \\ Japan Atomic Energy Research Institute, Tokai, Ibaraki
}

\begin{abstract}
Rapid methods of neutron activation analysis make it possible to detect easily gamma-emitting, short-lived nuclides using multichannel gamma spectrometers. Because neutrons from reactors - which are widely used in neutron activation - exhibit an extremely broad energy range from zero to at least $15 \mathrm{MeV}^{11}$, various types of neutron reactions are likely to occur simultaneously. For this reason, a particular reaction one selects to use is associated in most cases with interfering reactions that produce the desired activation product from elements other than the one sought. To analyze an element in a sample it is therefore necessary to consider all of these interfering reactions.

The table presented here shows original elements for these interfering reactions. The data have been collected from Table of Isotopes $^{2)}$ and the Rohrmann Chart ${ }^{3}$. The table is based on an original table of ShortLived Nuclides Formed by Neutron Activation $^{4}$.

Radioactive nuclides that emit only $\beta^{-}$or $\alpha$ particles were not listed in the table because they cannot be detected with gamma spectrometers.
\end{abstract}

Type of reaction To indicate the type of reaction responsible for the activity, numbers are given in parenthesis following each ele- ment. These numbers correspond to the following reactions :
1: $n, \gamma$
5: $n, 2 n$
9: $n,{ }^{3} \mathrm{He}$
2: $n, n^{\prime}$
6: $n, \alpha n$
10: $n, 2 p$
3: $n, p$
7: $n, p n$
4: $n, \alpha$
8: $n, t$

Energies and intensities Quantities enclosed in square brackets are calculated or inferred, not measured.

The intensities of radiations when expressed as percentages without other qualifications refer to percentages of the total decay events. A number following the dagger $(\dagger)$ symbol is the relative intensity for the particular mode of decay beside which the + appears.

\section{References}

1) D.J. Hughes: Nucleonics, 11 (1)30 (1953)

2) C. M. Lederer, J.M. Hollander, I. Perlman: "Table of Isotopes. Sixth Edition", John Wiley \& Sons, Inc. (1967)

3) C.A. Rohrmann: "Chart of the Nuclides Emphasizing Nuclear Power By-product Elements and Isotopes for Large Scale Applications", Battele-Northwest, Richland, Washington 99352, U.S.A., May (1969)

4) M. Okada: Nucleonics, 22,(8) 110 (1964) 


\begin{tabular}{|c|c|c|c|}
\hline $\begin{array}{l}\text { Element and } \\
\text { type of reaction }\end{array}$ & $\begin{array}{l}\text { Nuclide } \\
\text { formed }\end{array}$ & $\begin{array}{l}\text { Half } \\
\text { life }\end{array}$ & $\begin{array}{l}\text { Energies }(\mathrm{MeV}) \text { and intensities of } \gamma^{-} \text {, } \\
\mathrm{X}-\text { and annihilation radiations }\end{array}$ \\
\hline $\mathrm{Os}(7)$ & ${ }^{183 m} \mathrm{Re}$ & $1.0 \mathrm{~ms}$ & $0.194,0.11-0.30$ \\
\hline $\mathrm{Ru}(1,5)$ & ${ }^{97 m} \mathrm{Ru}$ & $1.8 \mathrm{~ms}$ & 0.227 \\
\hline $\mathrm{Bi}(5)$ & ${ }^{208 m} \mathrm{Bi}$ & $2.7 \mathrm{~ms}$ & $0.92(+100), 0.51(+90)$ \\
\hline $\mathrm{Pb}(1,5)$ & ${ }^{205 m} \mathrm{~Pb}$ & $4 \mathrm{~ms}$ & {$[0.703],[0.988],[1.04], \cdots$} \\
\hline $\mathrm{W}(2), \operatorname{Os}(6)$ & ${ }^{180 m} \mathrm{~W}$ & $5.2 \mathrm{~ms}$ & $0.10,0.15-0.5$ \\
\hline $\operatorname{Mo}(8)$ & ${ }^{90 m_{2}} \mathrm{Nb}$ & $0.010 \mathrm{~s}$ & 0.257 \\
\hline $\mathrm{C}(10)$ & ${ }^{12} \mathrm{Be}$ & $0.0114 \mathrm{~s}$ & $?$ \\
\hline $\mathrm{Y}(5), \operatorname{Zr}(8)$ & ${ }^{88 m} \mathrm{Y}$ & $0.014 \mathrm{~s}$ & $0.23,0.45$ \\
\hline $\operatorname{Pt}(7)$ & ${ }^{189 m} \operatorname{Ir}$ & $0.014 \mathrm{~s}$ & $0.072,0.114,0.187$ \\
\hline $\operatorname{As}(2), \operatorname{Se}(7,8), \operatorname{Br}(6)$ & ${ }^{75 m} \mathrm{As}$ & $0.017 \mathrm{~s}$ & $0.304(95 \%), \quad 0.280(<1 \%)$ \\
\hline $\mathrm{C}(3), \mathrm{N}(9,10)$ & ${ }^{13} \mathrm{~B}$ & $0.019 \mathrm{~s}$ & 3. $68(7 \%)$ \\
\hline $\mathrm{Na}(1), \operatorname{Mg}(3,7,8), \mathrm{Al}(4)$ & ${ }^{24 m} \mathrm{Na}$ & $0.020 \mathrm{~s}$ & 0.472 \\
\hline $\mathrm{Ge}(1,5), \mathrm{Se}(4)$ & ${ }^{71 m} \mathrm{Ge}$ & $0.020 \mathrm{~s}$ & 0.175 \\
\hline $\mathrm{B}(1), \mathrm{C}(3,7), \mathrm{N}(4,9)$ & ${ }^{12} \mathrm{~B}$ & $0.0203 \mathrm{~s}$ & $4.43(1.3 \%)$ \\
\hline $\mathrm{Ga}(1,5), \mathrm{Ge}(3,8)$ & ${ }^{70 m} \mathrm{Ga}$ & $0.021 \mathrm{~s}$ & 0.18 \\
\hline $\operatorname{Ir}(1), \operatorname{Pt}(3,7,8), \operatorname{Au}(4)$ & ${ }^{194 m} \operatorname{Ir}$ & $0.032 \mathrm{~s}$ & $\leq 0.10$ \\
\hline $\mathrm{Ga}(1), \mathrm{Ge}(3,7,8), \operatorname{As}(4)$ & ${ }^{72 m} \mathrm{Ga}$ & $0.036 \mathrm{~s}$ & $\leq 0.099$ \\
\hline $\operatorname{In}(5), \operatorname{Sn}(3,8)$ & ${ }^{112 m_{2}} \operatorname{In}$ & $0.042 \mathrm{~s}$ & 0.31 \\
\hline $\mathrm{Yb}(1,5), \operatorname{Lu}(3,7), \operatorname{Hf}(4,6,9,10)$ & ${ }^{175} \mathrm{Yb}$ & $0.067 \mathrm{~s}$ & $0.513[63 \%]$ \\
\hline $\mathrm{Be}(10)$ & ${ }^{8} \mathrm{He}$ & $0.122 \mathrm{~s}$ & $0.98(88 \%)$ \\
\hline $\mathrm{Be}(3), \mathrm{B}(9,10)$ & ${ }^{9} \mathrm{Li}$ & $0.17 \mathrm{~s}$ & $?$ \\
\hline $\mathrm{Ti}(3), \mathrm{V}(10)$ & ${ }^{50 m} \mathrm{Sc}$ & $0.35 \mathrm{~s}$ & $\begin{array}{l}0.258 \\
\text { daughter radiations from }{ }^{50} \mathrm{Sc}\end{array}$ \\
\hline $\mathrm{Ba}(1,2,5), \mathrm{La}(8), \mathrm{Ce}(6,9)$ & ${ }^{136 m} \mathrm{Ba}$ & $0.37 \mathrm{~s}$ & $\begin{array}{l}1.05(100 \%), 0.818(100 \%), \text { Ba X-rays, } \\
\quad 0.164(40 \%)\end{array}$ \\
\hline $\mathrm{Ge}(1,2,5), \operatorname{As}(8), \operatorname{Se}(4,10)$ & ${ }^{73 m} \mathrm{Ge}$ & $0.53 \mathrm{~s}$ & Ge X-rays, $0.054(9 \%)$ \\
\hline $\mathrm{O}(3), \mathrm{F}(10)$ & ${ }^{18} \mathrm{~N}$ & $0.63 \mathrm{~s}$ & $\begin{array}{l}1.98(100 \%), 1.65(59 \%), 0.82(59 \%) \\
\quad 2.47(41 \%)\end{array}$ \\
\hline $\mathrm{O}(9,10)$ & ${ }^{16} \mathrm{C}$ & $0.74 \mathrm{~s}$ & 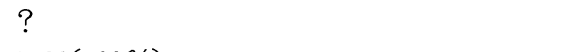 \\
\hline $\mathrm{Cl}(1), \operatorname{Ar}(3,8), \mathrm{K}(4,9,10)$ & ${ }^{38 m} \mathrm{Cl}$ & $0.74 \mathrm{~s}$ & $\begin{array}{l}0.66(100 \%) \\
\text { daughter radiations from }{ }^{38} \mathrm{Cl}\end{array}$ \\
\hline $\mathrm{Pb}(1,2,5), \mathrm{Bi}(8)$ & ${ }^{207 m} \mathrm{~Pb}$ & $0.80 \mathrm{~s}$ & $0.570(98 \%), \quad 1.064(83 \%)$ \\
\hline $\operatorname{Zr}(2,5), \operatorname{Mo}(6,9)$ & ${ }^{90 m} \mathrm{Zr}$ & $0.81 \mathrm{~s}$ & $\begin{array}{l}\mathrm{Zr} \text { X-rays, } 2.32(86 \%), 2.18(14 \%), 0.133 \\
\quad(4 \%)\end{array}$ \\
\hline $\mathrm{Li}(1), \mathrm{Be}(7), \mathrm{B}(4,9)$ & ${ }^{8} \mathrm{Li}$ & $0.84 \mathrm{~s}$ & $?$ \\
\hline $\mathrm{Ca}(5)$ & ${ }^{39} \mathrm{Ca}$ & $0.87 \mathrm{~s}$ & $0.511(200 \%)$ \\
\hline $\mathrm{K}(5), \mathrm{Ca}(8)$ & ${ }^{38 m} \mathrm{~K}$ & $0.95 \mathrm{~s}$ & $0.511(200 \%)$ \\
\hline $\operatorname{Mg}(3), \operatorname{Al}(10)$ & ${ }^{26} \mathrm{Na}$ & $1.0 \mathrm{~s}$ & $1.82(100 \%)$ \\
\hline $\operatorname{Er}(7)$ & ${ }^{163 m} \mathrm{Ho}$ & $1.1 \mathrm{~s}$ & $0.305[\approx 80 \%]$ \\
\hline $\mathrm{Cl}(5), \operatorname{Ar}(8)$ & ${ }^{34} \mathrm{Cl}$ & $1.56 \mathrm{~s}$ & $0.511(200 \%)$ \\
\hline $\operatorname{Ar}(5)$ & ${ }^{35} \mathrm{Ar}$ & $1.83 \mathrm{~s}$ & $0.511(200 \%), \quad 1.22(5 \%), \quad 1.76(2 \%)$ \\
\hline $\operatorname{In}(1), \operatorname{Sn}(3,7,8)$ & $116 m_{2} \operatorname{In}$ & $2.16 \mathrm{~s}$ & In X-rays, $0.164[\approx 40 \%]$ \\
\hline $\operatorname{Er}(1,2,5), \operatorname{Tm}(8), \operatorname{Yb}(4,6,10)$ & ${ }^{167 m} \mathrm{Er}$ & $2.3 \mathrm{~s}$ & Er X-rays, $0.208(43 \%)$ \\
\hline$N(3), O(4,9,10)$ & ${ }^{15} \mathrm{C}$ & $2.4 \mathrm{~s}$ & $5.299(68 \%)$ \\
\hline $\operatorname{In}(1,5), \operatorname{Sn}(3,7,8)$ & ${ }^{114} m_{2} \operatorname{In}$ & $2.5 \mathrm{~s}$ & 0.150 \\
\hline $\mathrm{S}(5)$ & ${ }^{31} \mathrm{~S}$ & $2.7 \mathrm{~s}$ & $0.511(200 \%), \quad 1.27(1.1 \%)$ \\
\hline $\operatorname{Sn}(3,8), \operatorname{Sb}(4,10)$ & ${ }^{120} \mathrm{In}$ & $3.2 \mathrm{~s}$ & $1.171(15 \%)$ \\
\hline
\end{tabular}




\begin{tabular}{|c|c|c|c|}
\hline $\begin{array}{l}\text { Element and } \\
\text { type of reaction }\end{array}$ & $\begin{array}{l}\text { Nuclide } \\
\text { formed }\end{array}$ & $\begin{array}{l}\text { Half } \\
\text { life }\end{array}$ & $\begin{array}{l}\text { Energies }(\mathrm{MeV}) \text { and intensities of } \gamma^{-}, \\
\mathrm{X}-\text { and annihilation radiations }\end{array}$ \\
\hline $\mathrm{Si}(3), P(10)$ & ${ }^{30} \mathrm{Al}$ & $3.3 \mathrm{~s}$ & $2.23(61 \%),[1.27(46 \%)], 3.51(39 \%)$ \\
\hline $\operatorname{Sn}(3)$ & ${ }^{124} \mathrm{In}$ & $\approx 3.6 \mathrm{~s}$ & $1.13\left(\dagger_{10}\right), 3.21\left(t^{3}\right), 0.99\left(\dagger^{3}\right)$ \\
\hline $\mathrm{Ne}(3), \mathrm{Na}(10)$ & ${ }^{22} \mathrm{~F}$ & $4.0 \mathrm{~s}$ & $1.28(100 \%), 2.06(67 \%)$ \\
\hline $\mathrm{O}(3,7), \mathrm{F}(9)$ & ${ }^{17} \mathrm{~N}$ & $4.16 \mathrm{~s}$ & $0.87(3 \%), 2.19(0.5 \%)$ \\
\hline $\operatorname{Si}(5)$ & ${ }^{27} \mathrm{Si}$ & $4.2 \mathrm{~s}$ & $0.511(200 \%)$ \\
\hline $\mathrm{Hf}(1,2,5), \quad \mathrm{Ta}(8), \mathrm{W}(6,9)$ & ${ }^{178 m} \mathrm{Hf}$ & $4.3 \mathrm{~s}$ & $\begin{array}{l}\text { Hf X-rays, } 0.427(97 \%), 0.326(94 \%) \\
\quad 0.214(75 \%), 0.089(54 \%), 0.093(14 \%)\end{array}$ \\
\hline $\mathrm{Ne}(3,7), \mathrm{Na}(9)$ & ${ }^{21} \mathrm{~F}$ & 4. $4 \mathrm{~s}$ & $0.350(\dagger 100), 1.38(\dagger 13)$ \\
\hline $\operatorname{Cd}(3,8), \operatorname{In}(10)$ & ${ }^{114} \mathrm{Ag}$ & $4.5 \mathrm{~s}$ & 0.57 \\
\hline $\operatorname{Br}(2), \operatorname{Kr}(7)$ & ${ }^{79 m} \mathrm{Br}$ & $4.8 \mathrm{~s}$ & {$[\mathrm{Br}$ X-rays], 0.21} \\
\hline $\operatorname{Ir}(2), \operatorname{Pt}(7)$ & ${ }^{191 m} \mathrm{Ir}$ & $4.9 \mathrm{~s}$ & Ir X-rays, $0.129(25 \%)$ \\
\hline $\operatorname{Ru}(3,8), \operatorname{Rh}(10)$ & ${ }^{102} \mathrm{Tc}$ & $5 s$ & $?$ \\
\hline $\operatorname{Pd}(3)$ & ${ }^{110} \mathrm{Rh}$ & $5 \mathrm{~s}$ & $0.374[\approx 14 \%]$ \\
\hline $\operatorname{Sn}(3,7,8), \operatorname{Sb}(4)$ & ${ }^{118}$ In & $5.1 \mathrm{~s}$ & $1.230(15 \%)$ \\
\hline $\mathrm{W}(1,2,5), \operatorname{Re}(8), \operatorname{Os}(4,6,10)$ & ${ }^{183 m} \mathrm{~W}$ & $5.3 \mathrm{~s}$ & $\begin{array}{l}\text { W X-rays, } 0.108(19 \%), 0.053(11 \%) \\
0.099(9 \%), 0.046(8 \%), 0.160(6 \%), \cdots\end{array}$ \\
\hline $\mathrm{Pb}(5)$ & ${ }^{203 m} \mathrm{~Pb}$ & $6.1 \mathrm{~s}$ & $\mathrm{~Pb} \mathrm{X}$-rays, $0.825(70 \%)$ \\
\hline $\operatorname{Os}(3), \operatorname{Ir}(10)$ & ${ }^{192} \mathrm{Re}$ & $6.2 \mathrm{~s}$ & $0.20,0.29,0.37,0.48,0.57$ \\
\hline $\mathrm{Al}(5), \mathrm{Si}(8)$ & ${ }^{26 m} \mathrm{Al}$ & $6.4 \mathrm{~s}$ & $0.511(200 \%)$ \\
\hline $\mathrm{Yb}(1), \operatorname{Hf}(4,9,10)$ & ${ }^{177 m} \mathrm{Yb}$ & $6.5 \mathrm{~s}$ & Yb X-rays, $0.104(65 \%), 0.228(13 \%)$ \\
\hline$N(1), O(3,7,8), F(4)$ & ${ }^{16} \mathrm{~N}$ & $7.2 \mathrm{~s}$ & $6.13(69 \%), 7.11(5 \%), 2.75(1 \%)$ \\
\hline $\operatorname{Au}(2), \operatorname{Hg}(7,8)$ & ${ }^{197 m} \mathrm{Au}$ & $7.2 \mathrm{~s}$ & Au X-rays, $0.279(75 \%), 0.130(8 \%)$ \\
\hline $\mathrm{Sn}(3,8), \mathrm{Sb}(10)$ & ${ }^{122} \operatorname{In}$ & $7.5 \mathrm{~s}$ & $0.99,1.14$ \\
\hline $\operatorname{As}(5), \operatorname{Se}(3,8)$ & ${ }^{74 m} \mathrm{As}$ & $8.0 \mathrm{~s}$ & 0.283 \\
\hline $\operatorname{Mo}(7)$ & ${ }^{99} \mathrm{Nb}$ & $10 \mathrm{~s}$ & $?$ \\
\hline $\operatorname{Sn}(7)$ & ${ }^{123} \mathrm{In}$ & $10 \mathrm{~s}$ & 1.1 \\
\hline $\mathrm{Tb}(5), \operatorname{Dy}(3,8)$ & ${ }^{158 m} \mathrm{~Tb}$ & $11 \mathrm{~s}$ & Tb X-rays, $0.110(0.5 \%)$ \\
\hline $\mathrm{F}(1), \mathrm{Ne}(3,7,8), \mathrm{Na}(4)$ & ${ }^{20} \mathrm{~F}$ & $11.4 \mathrm{~s}$ & 1. $63(100 \%)$ \\
\hline $\mathrm{Yb}(2), \operatorname{Lu}(3), \operatorname{Hf}(4,6,9,10)$ & ${ }^{176 m} \mathrm{Yb}$ & $12 \mathrm{~s}$ & Yb X-rays, $0.19,0.29,0.39$ \\
\hline $\operatorname{Mg}(5)$ & ${ }^{23} \mathrm{Mg}$ & $12.1 \mathrm{~s}$ & $0.511(200 \%), \quad 0.44(9 \%)$ \\
\hline $\mathrm{S}(3,8), \mathrm{Cl}(4,10)$ & ${ }^{34} \mathrm{P}$ & $12.4 \mathrm{~s}$ & $2.13(25 \%), 4.0(0.2 \%)$ \\
\hline $\operatorname{Kr}(1,5), \operatorname{Sr}(4)$ & ${ }^{81 m} \mathrm{Kr}$ & $13 \mathrm{~s}$ & Kr X-rays, $0.190(65 \%)$ \\
\hline $\mathrm{B}(3), \mathrm{C}(9,10)$ & ${ }^{11} \mathrm{Be}$ & $13.6 \mathrm{~s}$ & $\begin{array}{l}2.12(32 \%), 6.79(4.4 \%), 5.85(2.4 \%), 4.67 \\
\quad(2.1 \%), 7.99(1.7 \%)\end{array}$ \\
\hline $\mathrm{Ne}(9,10)$ & ${ }^{20} \mathrm{O}$ & $14 \mathrm{~s}$ & $1.06(100 \%)$ \\
\hline $\operatorname{In}(1), \operatorname{Sn}(3,7,8)$ & ${ }^{116} \operatorname{In}$ & $14 \mathrm{~s}$ & $1.293(1.2 \%), 0.434(0.12 \%), 0.95(0.1 \%)$ \\
\hline $\operatorname{Pt}(1), \operatorname{Hg}(4,9,10)$ & ${ }^{199 m} \mathrm{Pt}$ & $14.1 \mathrm{~s}$ & Pt X-rays, $0.393(90 \%)$ \\
\hline $\operatorname{Se}(3,8), \operatorname{Br}(10)$ & ${ }^{80} \mathrm{As}$ & $15.3 \mathrm{~s}$ & $\begin{array}{l}0.666(42 \%), 1.64(4 \%), 1.22(4 \%), 1.77 \\
\quad(1.7 \%), 0.8(1.4 \%, \text { complex })\end{array}$ \\
\hline $\mathrm{Y}(2), \operatorname{Zr}(7,8), \mathrm{Nb}(6)$ & ${ }^{89 m} \mathrm{Y}$ & $16 \mathrm{~s}$ & $0.91(99 \%)$ \\
\hline $\operatorname{Ru}(3,7,8), \quad \operatorname{Rh}(4)$ & ${ }^{100} \mathrm{Tc}$ & $17 \mathrm{~s}$ & $\begin{array}{l}0.540 \text { (strong), } 0.60 \text { (strong), } 0.71,0.81 \\
0.89,1.01,1.31,1.49,1.8\end{array}$ \\
\hline $\operatorname{Pd}(3,8), \operatorname{Ag}(10)$ & ${ }^{108} \mathrm{Rh}$ & $17 \mathrm{~s}$ & $0.434(43 \%), 0.62(22 \%), 0.51(10 \%$, complex $)$ \\
\hline $\mathrm{Ne}(5)$ & ${ }^{19} \mathrm{Ne}$ & $17.5 \mathrm{~s}$ & $0.511(200 \%)$ \\
\hline $\mathrm{Ca}(7)$ & ${ }^{47} \mathrm{~K}$ & $17.5 \mathrm{~s}$ & $2.0(84 \%), 2.6(15 \%)$ \\
\hline $\mathrm{Se}(1,5), \operatorname{Br}(8), \operatorname{Kr}(4,10)$ & ${ }^{77 m} \mathrm{Se}$ & $17.5 \mathrm{~s}$ & Se X-rays, $0.161(50 \%)$ \\
\hline $\mathrm{Hf}(1,2,5), \mathrm{Ta}(7,8), \mathrm{W}(4,6,10)$ & ${ }^{179 m} \mathrm{Hf}$ & $18.6 \mathrm{~s}$ & Hf X-rays, $0.217(94 \%), \cdots$ \\
\hline $\mathrm{Sc}(1), \quad \operatorname{Ti}(3,7,8), \mathrm{V}(6)$ & ${ }^{46 m} \mathrm{Sc}$ & $20 \mathrm{~s}$ & {$[$ Sc X-rays $], 0.142$} \\
\hline
\end{tabular}




\begin{tabular}{|c|c|c|c|}
\hline $\begin{array}{l}\text { Element and } \\
\text { type of reaction }\end{array}$ & $\begin{array}{l}\text { Nuclide } \\
\text { formed }\end{array}$ & $\begin{array}{l}\text { Half } \\
\text { life }\end{array}$ & $\begin{array}{l}\text { Energies }(\mathrm{MeV}) \text { and intensities of } \gamma^{-}, \\
\mathrm{X}-\text { and annihilation radiations }\end{array}$ \\
\hline $\operatorname{Pd}(1,5), \operatorname{Ag}(3,8), \operatorname{Cd}(4,6,10)$ & ${ }^{107 m} \mathrm{Pd}$ & $22 \mathrm{~s}$ & Pd X-rays, 0.21 \\
\hline $\operatorname{Mo}(8)$ & ${ }^{90 m_{1}} \mathrm{Nb}$ & $24 \mathrm{~s}$ & Nb X-rays, $0.122(71 \%)$ \\
\hline $\operatorname{Ag}(1), \operatorname{Cd}(3,7,8), \operatorname{In}(4)$ & ${ }^{110} \mathrm{Ag}$ & $24.4 \mathrm{~s}$ & $0.658(4.5 \%)$ \\
\hline $\mathrm{Hg}(3,8), \quad \mathrm{Tl}(4,10)$ & ${ }^{202} \mathrm{Au}$ & $\approx 25 \mathrm{~s}$ & $\gamma ?$ \\
\hline $\mathrm{O}(1), \mathrm{F}(3), \mathrm{Ne}(4,9,10)$ & ${ }^{19} \mathrm{O}$ & $29 \mathrm{~s}$ & $0.197(97 \%), 1.37(59 \%), 1.44[2.5 \%]$ \\
\hline $\mathrm{Xe}(10)$ & ${ }^{135} \mathrm{Te}$ & $29 \mathrm{~s}$ & $?$ \\
\hline $\operatorname{Zn}(3,8), \quad \mathrm{Ga}(4,10)$ & ${ }^{68} \mathrm{Cu}$ & $30 \mathrm{~s}$ & $1.078(95 \%), 0.80(17 \%), 1.88(5 \%), 1.24(3 \%)$ \\
\hline $\operatorname{Pd}(3,8), \operatorname{Ag}(4,10)$ & ${ }^{106} \mathrm{Rh}$ & $30 \mathrm{~s}$ & $\begin{array}{l}0.512(21 \%), 0.622(11 \% \text {, doublet }), 1.05 \\
(1.5 \%, \text { doublet }), 1.13(0.5 \% \text {, doublet }) \\
1.55(0.2 \%)\end{array}$ \\
\hline $\operatorname{Pd}(7)$ & ${ }^{109} \mathrm{Rh}$ & $30 \mathrm{~s}$ & $0.49, \quad 0.31$ \\
\hline $\mathrm{Sn}(7), \mathrm{Sb}(9)$ & ${ }^{121} \mathrm{In}$ & $30 \mathrm{~s}$ & 0.94 \\
\hline $\mathrm{Gd}(4,10)$ & ${ }^{157} \mathrm{Sm}$ & $\approx 30 \mathrm{~s}$ & 0.57 \\
\hline $\mathrm{Hg}(7)$ & ${ }^{195 m} \mathrm{Au}$ & $31 \mathrm{~s}$ & Au X-rays, $0.261(77 \%)$ \\
\hline $\mathrm{Ge}(3)$ & ${ }^{76} \mathrm{Ga}$ & $32 \mathrm{~s}$ & $0.563,0.95,1.12$ \\
\hline $\operatorname{Sn}(7)$ & ${ }^{123} \operatorname{In}$ & $36 \mathrm{~s}$ & $?$ \\
\hline $\mathrm{Ne}(1), \mathrm{Na}(3), \operatorname{Mg}(4,9,10)$ & ${ }^{23} \mathrm{Ne}$ & $37.6 \mathrm{~s}$ & $\begin{array}{l}0.439(33 \%), 1.64(0.9 \%), 2.07(0.1 \%) \\
\quad 2.99(0.03 \%), \cdots\end{array}$ \\
\hline $\operatorname{Kr}(10)$ & ${ }^{85} \mathrm{Se}$ & $39 \mathrm{~s}$ & $?$ \\
\hline $\operatorname{Ag}(2), \operatorname{Cd}(7,8), \operatorname{In}(6)$ & ${ }^{109 m} \mathrm{Ag}$ & $40 \mathrm{~s}$ & Ag X-rays, $0.088(5 \%)$ \\
\hline $\operatorname{Sm}(8)$ & ${ }^{142} \mathrm{Pm}$ & $40 \mathrm{~s}$ & $0.511(190 \%), \mathrm{Nd} \mathrm{X}$-rays \\
\hline $\operatorname{Rh}(1), \operatorname{Pd}(3,7,8), \operatorname{Ag}(4)$ & ${ }^{104} \mathrm{Rh}$ & $43 \mathrm{~s}$ & $\mathrm{Ru}$ X-rays, $0.56(2.0 \%), 1.24(0.13 \%), \cdots$ \\
\hline $\operatorname{Nd}(9)$ & ${ }^{148} \mathrm{Ce}$ & $\approx 43 \mathrm{~s}$ & 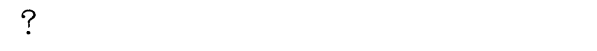 \\
\hline $\operatorname{Er}(3)$ & ${ }^{170} \mathrm{Ho}$ & $44 \mathrm{~s}$ & 0.43 \\
\hline $\operatorname{Ag}(2), \operatorname{Cd}(7)$ & ${ }^{107 m} \mathrm{Ag}$ & $44.3 \mathrm{~s}$ & Ag X-rays, $0.094(5 \%)$ \\
\hline $\operatorname{Pd}(3,7), \operatorname{Ag}(6,9)$ & ${ }^{105 m} \mathrm{Rh}$ & $45 \mathrm{~s}$ & Rh X-rays, 0.129 \\
\hline $\mathrm{Cd}(10)$ & ${ }^{115} \mathrm{Pd}$ & $45 \mathrm{~s}$ & $?$ \\
\hline $\operatorname{Sn}(3,8), \operatorname{Sb}(4,10)$ & ${ }^{120} \mathrm{In}$ & $46 \mathrm{~s}$ & $\begin{array}{l}1.171(15 \%), 1.02(9 \%), 0.86(5 \%), 1.28 \\
\quad(2 \%), \cdots\end{array}$ \\
\hline $\mathrm{Yb}(1,5)$ & ${ }^{169 m} \mathrm{Yb}$ & $46 \mathrm{~s}$ & Yb L X-rays \\
\hline $\operatorname{Zr}(10)$ & ${ }^{95} \mathrm{Sr}$ & $\approx 48 \mathrm{~s}$ & $?$ \\
\hline $\mathrm{Ge}(1,5), \operatorname{As}(3), \operatorname{Se}(4,9,10)$ & ${ }^{75 m} \mathrm{Ge}$ & $49 \mathrm{~s}$ & Ge X-rays, $0.139(34 \%)$ \\
\hline $\operatorname{Zn}(4,10)$ & ${ }^{67} \mathrm{Ni}$ & $50 \mathrm{~s}$ & $0.90(51 \%$, doublet $), 1.26(15 \%)$ \\
\hline $\mathrm{Ru}(7)$ & ${ }^{103} \mathrm{Tc}$ & $50 \mathrm{~s}$ & $0.135\left(\dagger^{\dagger} 17\right), \quad 0.21(\dagger 10), \quad 0.35$ \\
\hline $\operatorname{Pd}(7)$ & ${ }^{109 m} \mathrm{Rh}$ & $50 \mathrm{~s}$ & 0.11 \\
\hline $\operatorname{Pt}(3)$ & ${ }^{198} \mathrm{Ir}$ & $50 \mathrm{~s}$ & 0.78 \\
\hline $\operatorname{Ge}(1), \operatorname{Se}(4,10)$ & ${ }^{77 m} \mathrm{Ge}$ & $54 \mathrm{~s}$ & Ge X-rays, $0.215(21 \%), 0.159(12 \%)$ \\
\hline $\operatorname{Kr}(3), \quad \operatorname{Rb}(10)$ & ${ }^{86} \mathrm{Br}$ & $54 \mathrm{~s}$ & 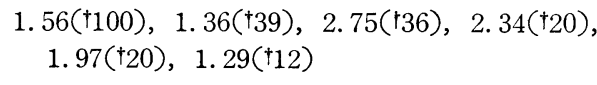 \\
\hline $\mathrm{Cr}(3), \mathrm{Mn}(10)$ & ${ }^{54} \mathrm{~V}$ & $55 \mathrm{~s}$ & $0.99(100 \%), \quad 0.84(100 \%), 2.21[100 \%]$ \\
\hline $\operatorname{Kr}(1,5)$ & ${ }^{79 m} \mathrm{Kr}$ & $55 \mathrm{~s}$ & Kr X-rays, 0.127 \\
\hline $\mathrm{Xe}(1,5)$ & ${ }^{125 m} \mathrm{Xe}$ & $55 \mathrm{~s}$ & Xe X-rays, $0.075,0.111$ \\
\hline $\mathrm{Ce}(1,5), \operatorname{Pr}(8), \operatorname{Nd}(4,6)$ & ${ }^{139 m} \mathrm{Ce}$ & $55 \mathrm{~s}$ & Ce X-rays, $0.746(93 \%)$ \\
\hline $\mathrm{Hg}(7), \quad \mathrm{Tl}(9)$ & ${ }^{203} \mathrm{Au}$ & $55 \mathrm{~s}$ & 0.69 \\
\hline $\operatorname{Mg}(3,7), \operatorname{Al}(9)$ & ${ }^{25} \mathrm{Na}$ & $60 \mathrm{~s}$ & $0.98(15 \%), 0.58(14 \%), 0.39(14 \%), 1.61(6 \%)$ \\
\hline $\operatorname{Mo}(3,7)$ & ${ }^{97 m} \mathrm{Nb}$ & $\approx 60 \mathrm{~s}$ & $0.747(98 \%)$ \\
\hline $\mathrm{Rb}(1,5), \operatorname{Sr}(3,7,8), \mathrm{Y}(4)$ & ${ }^{86 m} \mathrm{Rb}$ & $62 \mathrm{~s}$ & $\mathrm{Rb} \mathrm{X}$-rays, 0.56 \\
\hline $\operatorname{Nd}(5), \operatorname{Sm}(4)$ & ${ }^{141 m} \mathrm{Nd}$ & $63 \mathrm{~s}$ & $0.755[90 \sim 100 \%]$ \\
\hline
\end{tabular}




\begin{tabular}{|c|c|c|c|}
\hline $\begin{array}{l}\text { Element and } \\
\text { type of reaction }\end{array}$ & $\begin{array}{l}\text { Nuclide } \\
\text { formed }\end{array}$ & $\begin{array}{l}\text { Half } \\
\text { life }\end{array}$ & $\begin{array}{l}\text { Energies }(\mathrm{MeV}) \text { and intensities of } \gamma^{-} \text {, } \\
\mathrm{X}-\text { and annihilation radiations }\end{array}$ \\
\hline $\operatorname{Sm}(5)$ & ${ }^{143 m} \mathrm{Sm}$ & $64 \mathrm{~s}$ & 0.748 \\
\hline $\mathrm{Nd}(4,10)$ & ${ }^{147} \mathrm{Ce}$ & $65 \mathrm{~s}$ & $?$ \\
\hline $\mathrm{Fe}(3), \mathrm{Co}(10)$ & ${ }^{38} \mathrm{Mn}$ & $\approx 66 \mathrm{~s}$ & $\begin{array}{l}0.36,0.41,0.52,0.57,0.82,1.0,1.25 \\
\begin{array}{l}1.4,1.6,2.2,2.8\end{array}\end{array}$ \\
\hline $\operatorname{Mo}(5)$ & ${ }^{91 m} \mathrm{Mo}$ & $66 \mathrm{~s}$ & $\begin{array}{l}0.511[76 \%], \text { Mo X-rays, } 0.658(54 \%), \mathrm{Nb} \\
\text { X-rays, } 1.21(22 \%), 1.53(15 \%)\end{array}$ \\
\hline $\mathrm{Se}(1), \operatorname{Kr}(4,10)$ & ${ }^{83 m} \mathrm{Se}$ & $70 \mathrm{~s}$ & $\begin{array}{l}\text { 1. } 01\left(\dagger^{\dagger} 100, \text { complex }\right), 2.02(\dagger 40), 0.65(\dagger 20) \\
0.35(\dagger 16)\end{array}$ \\
\hline & & & daughter radiations from ${ }^{83} \mathrm{Br},{ }^{83 m} \mathrm{Kr}$ \\
\hline $\mathrm{U}(3,7)$ & ${ }^{234 m} \mathrm{~Pa}$ & $70.3 \mathrm{~s}$ & U LX-rays, $1.001(0.60 \%), 0.765(0.30 \%), \cdots$ \\
\hline $\mathrm{Cd}(3,7), \operatorname{In}(9)$ & ${ }^{113} \mathrm{Ag}$ & $\approx 72 \mathrm{~s}$ & $0.14,0.30,0.39,0.56,0.70$ \\
\hline $\operatorname{In}(1,5), \operatorname{Sn}(3,7,8)$ & ${ }^{114} \operatorname{In}$ & $72 \mathrm{~s}$ & Cd X-rays, $1.299(0.17 \%)$ \\
\hline $\operatorname{Cd}(3,7,8), \operatorname{In}(6,9)$ & ${ }^{111 m} \mathrm{Ag}$ & $74 \mathrm{~s}$ & [Ag X-rays], 0.065 \\
\hline $\operatorname{Sr}(8)$ & ${ }^{82} \mathrm{Rb}$ & $75 \mathrm{~s}$ & Kr X-rays, $0.511(192 \%), 0.777(9 \%), \cdots$ \\
\hline $\mathrm{Xe}(1,5), \mathrm{Ba}(4)$ & ${ }^{127 m} \mathrm{Xe}$ & $75 \mathrm{~s}$ & Xe X-rays, $0.125,0.175$ \\
\hline $\operatorname{Dy}(1), \operatorname{Ho}(3), \operatorname{Er}(4,9,10)$ & ${ }^{165 m_{1}} \mathrm{Dy}$ & $75.4 \mathrm{~s}$ & $\begin{array}{l}\text { Dy X-rays, } 0.108(3 \%), 0.514(1.8 \%), 0.362 \\
\quad(0.6 \%), 0.152(0.3 \%) \\
\text { daughter radiations from }{ }^{165} \mathrm{Dy}\end{array}$ \\
\hline $\operatorname{Zr}(9)$ & ${ }^{94} \mathrm{Sr}$ & $\approx 78 \mathrm{~s}$ & 1. $42(100 \%)$ \\
\hline $\mathrm{Xe}(3)$ & ${ }^{136} \mathrm{I}$ & $83 \mathrm{~s}$ & $\begin{array}{l}\text { 1. } 32(95 \% \text {, complex }), 2.3(19 \% \text {, complex }) \\
\begin{array}{l}0.39(19 \%), 0.27(18 \%), 0.20(12 \%) \\
2.63(10 \%), 2.8(8 \%), 3.2(5 \%)\end{array}\end{array}$ \\
\hline $\operatorname{Ar}(3), \mathrm{K}(10)$ & ${ }^{40} \mathrm{Cl}$ & $1.4 \mathrm{~m}$ & $2.83(+100), 1.46\left(\dagger^{\dagger} 100\right), 5.8,3.10$ \\
\hline $\mathrm{Cd}(4,10)$ & ${ }^{113} \mathrm{Pd}$ & $1.4 \mathrm{~m}$ & $\begin{array}{l}\text { no } \gamma \\
\text { daughter radiations from } 5.3 \mathrm{~h} \quad{ }^{113} \mathrm{Ag} \text { andl } \\
\quad 1.2 \mathrm{~m}{ }^{113} \mathrm{Ag}\end{array}$ \\
\hline $\operatorname{Ir}(1,5), \operatorname{Pt}(3,8)$ & ${ }^{192 m_{1}} \operatorname{Ir}$ & $1.4 \mathrm{~m}$ & $\begin{array}{l}\text { Ir L X-rays, } 0.317(0.008 \%), 0.058(0.005 \\
\quad \%), 0.612(0.003 \%)\end{array}$ \\
\hline $\mathrm{Sb}(1), \mathrm{Te}(3,7,8), \mathrm{I}(4)$ & $124 m_{1} \mathrm{Sb}$ & $1.55 \mathrm{~m}$ & $\begin{array}{l}\text { Sb L X-rays, } 0.644(20 \%), 0.603(20 \%) \\
\quad 0.505(20 \%)\end{array}$ \\
\hline $\mathrm{Ni}(3,8), \mathrm{Cu}(4,10)$ & ${ }^{62} \mathrm{Co}$ & $1.6 \mathrm{~m}$ & $\gamma$ rays observed \\
\hline$W(1,5), \operatorname{Re}(3,8), \operatorname{Os}(4,6,9,10)$ & ${ }^{185 m} \mathrm{~W}$ & $1.6 \mathrm{~m}$ & $\begin{array}{l}\text { W X-rays, } 0.17(† 100), 0.13(\uparrow 70), 0.100(\uparrow 16), \\
0.075(\dagger 8)\end{array}$ \\
\hline $\mathrm{Fe}(3,7), \mathrm{Co}(9)$ & ${ }^{57} \mathrm{Mn}$ & $1.7 \mathrm{~m}$ & 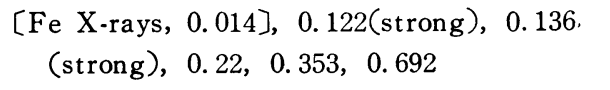 \\
\hline $\mathrm{Ti}(3), \mathrm{V}(10)$ & ${ }^{50} \mathrm{Sc}$ & $1.72 \mathrm{~m}$ & $1.55(100 \%), 1.12(100 \%), 0.520(100 \%)$ \\
\hline $\operatorname{Cr}(3,7), \operatorname{Mn}(9)$ & ${ }^{53} \mathrm{~V}$ & $2.0 \mathrm{~m}$ & $1.00[100 \%]$ \\
\hline $\mathrm{Ge}(7)$ & ${ }^{75} \mathrm{Ga}$ & $2 \mathrm{~m}$ & $\begin{array}{l}0.58(3 \%), 0.36 ?(1 \%) \\
\left.\text { [daughter radiations from }{ }^{75} \mathrm{Ge}\right]\end{array}$ \\
\hline $\operatorname{Nd}(3,8)$ & ${ }^{148} \mathrm{Pr}$ & $2.0 \mathrm{~m}$ & 0.30 \\
\hline $\mathrm{O}(5)$ & ${ }^{15} \mathrm{O}$ & $2.06 \mathrm{~m}$ & $0.511(200 \%)$ \\
\hline $\operatorname{Sn}(3,7), \operatorname{Sb}(6,9)$ & ${ }^{119} \mathrm{In}$ & $2.1 \mathrm{~m}$ & $0.82(95 \%), \cdots$ \\
\hline $\operatorname{Zr}(3)$ & ${ }^{96} \mathrm{Y}$ & $2.3 \mathrm{~m}$ & $0.7,1.0,1.5$ (complex) \\
\hline $\operatorname{Nd}(7)$ & ${ }^{149} \mathrm{Pr}$ & $2.3 \mathrm{~m}$ & $0.08,0.155,0.325,0.36, \quad 0.745$ \\
\hline $\mathrm{Al}(1), \mathrm{Si}(3,7,8), \mathrm{P}(4)$ & ${ }^{28} \mathrm{Al}$ & $2.31 \mathrm{~m}$ & $1.780(100 \%)$ \\
\hline $\mathrm{Zn}(1), \mathrm{Ga}(3), \mathrm{Ge}(4,9,10)$ & ${ }^{71} \mathrm{Zn}$ & $2.4 \mathrm{~m}$ & $\begin{array}{l}0.510(13 \%), 0.92(3 \%), 1.12(1.3 \%), 0.39 \\
\quad(1.3 \%), 0.120(0.9 \%)\end{array}$ \\
\hline
\end{tabular}




\begin{tabular}{|c|c|c|c|}
\hline $\begin{array}{l}\text { Element and } \\
\text { type of reaction }\end{array}$ & $\begin{array}{l}\text { Nuclide } \\
\text { formed }\end{array}$ & $\begin{array}{l}\text { Half } \\
\text { life }\end{array}$ & $\begin{array}{l}\text { Energies }(\mathrm{MeV}) \text { and intensities of } \gamma^{-} \text {, } \\
\mathrm{X}-\text { and annihilation radiations }\end{array}$ \\
\hline $\mathrm{Mo}(7)$ & ${ }^{99} \mathrm{Nb}$ & $2.4 \mathrm{~m}$ & $0.260(+1), 0.100(+1)$ \\
\hline $\operatorname{Ag}(1,5), \operatorname{Cd}(3,8)$ & ${ }^{108} \mathrm{Ag}$ & $2.42 \mathrm{~m}$ & $\begin{array}{c}\text { Pd X-rays, } 0.632(1.7 \%), 0.511(0.56 \%) \\
0.434(0.45 \%), 0.615(0.18 \%)\end{array}$ \\
\hline$P(5), S(8)$ & ${ }^{30} \mathrm{P}$ & $2.50 \mathrm{~m}$ & $0.511(200 \%), 2.23(0.5 \%)$ \\
\hline $\mathrm{Cd}(3)$ & ${ }^{116} \mathrm{Ag}$ & $2.5 \mathrm{~m}$ & $0.52,0.70$ \\
\hline $\operatorname{Sm}(3)$ & ${ }^{154} \mathrm{Pm}$ & $2.5 \mathrm{~m}$ & $?$ \\
\hline $\mathrm{Hg}(4,10)$ & ${ }^{201} \mathrm{Pt}$ & $2.5 \mathrm{~m}$ & $\begin{array}{l}0.15,0.23,1.76 \\
\text { daughter radiation from }{ }^{201} \mathrm{Au}\end{array}$ \\
\hline $\mathrm{Ba}(1,2,5), \mathrm{La}(7,8), \mathrm{Ce}(4,10)$ & ${ }^{137 m} \mathrm{Ba}$ & $2.55 \mathrm{~m}$ & Ba X-rays, $0.662(89 \%)$ \\
\hline $\operatorname{Sn}(4,10)$ & ${ }^{119} \mathrm{Cd}$ & $2.7 \mathrm{~m}$ & daughter radiations from ${ }^{119 m} \mathrm{In},{ }^{119} \mathrm{In}$ \\
\hline $\operatorname{Os}(3,8), \operatorname{Ir}(4,10)$ & ${ }^{190} \mathrm{Re}$ & $2.8 \mathrm{~m}$ & 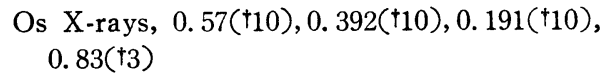 \\
\hline $\mathrm{Kr}(7), \mathrm{Rb}(9)$ & ${ }^{85} \mathrm{Br}$ & $3.0 \mathrm{~m}$ & $\begin{array}{l}\text { no } \gamma \\
\text { daughter radiations from }{ }^{85 m} \mathrm{Kr}\end{array}$ \\
\hline $\operatorname{Mo}(3)$ & ${ }^{100} \mathrm{Nb}$ & $3.0 \mathrm{~m}$ & $\begin{array}{c}0.53(\dagger 100, \text { complex }), 0.36(\uparrow 55), 0.45(\dagger 40) \\
0.140(\uparrow 10), 0.65,2.2,2.3,2.65,2.85\end{array}$ \\
\hline $\operatorname{Nd}(4,10)$ & ${ }^{145} \mathrm{Ce}$ & $3.0 \mathrm{~m}$ & $0.72,0.44, \cdots$ \\
\hline $\mathrm{Sn}(7), \mathrm{Sb}(9)$ & ${ }^{121} \mathrm{In}$ & $3.1 \mathrm{~m}$ & $?$ \\
\hline $\mathrm{Pb}(3), \mathrm{Bi}(10)$ & ${ }^{208} \mathrm{Tl}$ & $3.10 \mathrm{~m}$ & $\begin{array}{l}2.614(100 \%), 0.583(86 \%), 0.511(23 \%) \\
\quad 0.860(12 \%)\end{array}$ \\
\hline $\mathrm{Kr}(9)$ & ${ }^{84} \mathrm{Se}$ & $3.3 \mathrm{~m}$ & $?$ \\
\hline $\operatorname{Er}(3,8), \operatorname{Tm}(10)$ & ${ }^{168} \mathrm{Ho}$ & $3.3 \mathrm{~m}$ & 0.85 \\
\hline $\operatorname{Mg}(9,10)$ & ${ }^{24} \mathrm{Ne}$ & $3.38 \mathrm{~m}$ & $\begin{array}{l}0.472\left(100 \% \text {, with }{ }^{24 m} \mathrm{Na}\right), 0.88(8 \%) \\
\text { daughter radiations from }{ }^{24} \mathrm{Na}\end{array}$ \\
\hline $\operatorname{Pr}(5), \operatorname{Nd}(8)$ & ${ }^{140} \mathrm{Pr}$ & $3.39 \mathrm{~m}$ & $0.511(100 \%)$, Ce X-rays, $1.596(0.3 \%)$ \\
\hline $\mathrm{Te}(8)$ & ${ }^{118} \mathrm{Sb}$ & $3.5 \mathrm{~m}$ & $\begin{array}{l}0.511(150 \%) \text {, Sn X-rays, } 1.230(3 \% \text {, } \\
\text { doublet }), 0.83(0.4 \%)\end{array}$ \\
\hline $\mathrm{Xe}(8)$ & ${ }^{122} \mathrm{I}$ & $3.5 \mathrm{~m}$ & $0.511[130 \%]$, Te X-rays, $0.564,0.69,0.78$ \\
\hline $\operatorname{Gd}(1), \operatorname{Dy}(4,9,10)$ & ${ }^{161} \mathrm{Gd}$ & $3.7 \mathrm{~m}$ & $\begin{array}{l}\text { Tb X-rays, } 0.361(66 \%), 0.315(25 \%) \\
0.102(11 \%), 0.284(8 \%)\end{array}$ \\
\hline $\mathrm{V}(1), \operatorname{Cr}(3,7,8), \operatorname{Mn}(4)$ & ${ }^{52} \mathrm{~V}$ & $3.76 \mathrm{~m}$ & $1.434(100 \%)$ \\
\hline $\mathrm{Ba}(8)$ & ${ }^{128} \mathrm{Cs}$ & $3.8 \mathrm{~m}$ & $\begin{array}{c}0.511(110 \%), \mathrm{Xe} X \text {-rays, } 0.441(27 \%) \\
1.12(1 \%), 0.97(1 \%), 0.528,0.576\end{array}$ \\
\hline $\mathrm{Se}(1,5), \operatorname{Br}(3,8), \operatorname{Kr}(4,6,10)$ & ${ }^{79 m} \mathrm{Se}$ & $3.9 \mathrm{~m}$ & Se X-rays, $0.096(9 \%)$ \\
\hline $\mathrm{Xe}(1), \mathrm{Ba}(10)$ & ${ }^{137} \mathrm{Xe}$ & $3.9 \mathrm{~m}$ & $0.455(33 \%)$ \\
\hline $\mathrm{Te}(4,10)$ & ${ }^{127} \mathrm{Sn}$ & $4 \mathrm{~m}$ & $0.49(100 \%)$ \\
\hline $\operatorname{Zr}(5), \operatorname{Mo}(4)$ & ${ }^{89 m} \mathrm{Zr}$ & $4.18 \mathrm{~m}$ & $\mathrm{Zr}, \mathrm{Y} \mathrm{X}$-rays, $0.588(87 \%), 1.51(6 \%)$ \\
\hline $\operatorname{Kr}(7)$ & ${ }^{77 m} \mathrm{Br}$ & $4.2 \mathrm{~m}$ & [Br X-rays], 0.108 \\
\hline $\operatorname{Pd}(4,10)$ & ${ }^{107} \mathrm{Ru}$ & $4.2 \mathrm{~m}$ & $\begin{array}{l}0.195(14 \%), 0.86(7 \%), 1.29(4 \%), 1.03 \\
\quad(4 \%), 0.93(4 \%), \cdots \\
\text { daughter radiations from }{ }^{107} \mathrm{Rh}\end{array}$ \\
\hline $\mathrm{Sb}(1,5), \mathrm{Te}(3,7,8)$ & ${ }^{122 m} \mathrm{Sb}$ & $4.2 \mathrm{~m}$ & $\mathrm{Sb} \mathrm{X}$-rays, $0.061(50 \%), 0.075(17 \%)$ \\
\hline $\operatorname{Sn}(3,7,8), \operatorname{Sb}(4)$ & ${ }^{118} \mathrm{In}$ & $4.4 \mathrm{~m}$ & $\begin{array}{l}1.230(97 \%), 1.05(80 \%), 0.69(41 \%), 2.04 \\
\quad(3 \%)\end{array}$ \\
\hline $\operatorname{Er}(4,10)$ & ${ }^{167} \mathrm{Dy}$ & $4.4 \mathrm{~m}$ & $0.19,0.26,0.31,0.51,0.57$ \\
\hline $\operatorname{Rh}(1), \operatorname{Pd}(3,7,8), \operatorname{Ag}(4)$ & ${ }^{104 m} \mathrm{Rh}$ & $4.41 \mathrm{~m}$ & $\begin{array}{l}\text { Rh X-rays, } 0.051(47 \%), 0.097(2.6 \%) \\
\quad 0.078(2.5 \%), 0.77(0.24 \% \text { doublet }), 0.56 \\
\quad(0.18 \%)\end{array}$ \\
\hline
\end{tabular}




\begin{tabular}{|c|c|c|c|}
\hline $\begin{array}{l}\text { Element and } \\
\text { type of reaction }\end{array}$ & $\begin{array}{l}\text { Nuclide } \\
\text { formed }\end{array}$ & $\begin{array}{l}\text { Half } \\
\text { life }\end{array}$ & $\begin{array}{l}\text { Energies }(\mathrm{MeV}) \text { and intensities of } \gamma^{-}, \\
\mathrm{X}^{-} \text {and annihilation radiations }\end{array}$ \\
\hline $\operatorname{Ru}(3,8), \quad \operatorname{Rh}(10)$ & ${ }^{102} \mathrm{Tc}$ & $4.5 \mathrm{~m}$ & $0.47, \cdots$ \\
\hline $\operatorname{Pd}(9)$ & ${ }^{108} \mathrm{Ru}$ & $4.5 \mathrm{~m}$ & $\begin{array}{l}0.165(28 \%) \\
\text { daughter radiations from }{ }^{108} \mathrm{Rh}\end{array}$ \\
\hline $\operatorname{Pd}(1,5), \quad \operatorname{Ag}(3), \operatorname{Cd}(4,6,9,10)$ & ${ }^{109 m} \mathrm{Pd}$ & $4.7 \mathrm{~m}$ & Pd X-rays, $0.188(58 \%)$ \\
\hline $\mathrm{Pb}(3,7), \quad \mathrm{Bi}(9)$ & ${ }^{207} \mathrm{Tl}$ & $4.78 \mathrm{~m}$ & $0.897(0.16 \%)$ \\
\hline $\operatorname{Er}(7)$ & ${ }^{169} \mathrm{Ho}$ & $4.8 \mathrm{~m}$ & $\begin{array}{l}0.76(+30), 0.84\left(\dagger^{\dagger} 15\right), 0.15(\dagger 12), 0.68(+10) \\
\quad 0.92(\dagger 5), \cdots\end{array}$ \\
\hline $\operatorname{Hf}(3,7,8), \quad \mathrm{Ta}(4,9)$ & ${ }^{178} \mathrm{Lu}$ & $5 \mathrm{~m}$ & $0.090,0.22,0.33,0.43$ \\
\hline $\mathrm{S}(1), \mathrm{Cl}(3), \operatorname{Ar}(4,10)$ & ${ }^{37} \mathrm{~S}$ & $5.06 \mathrm{~m}$ & 3. $09(90 \%)$ \\
\hline $\mathrm{Cu}(1), \mathrm{Zn}(3,7,8), \mathrm{Ga}(4)$ & ${ }^{66} \mathrm{Cu}$ & $5.1 \mathrm{~m}$ & $1.039(9 \%), \cdots$ \\
\hline $\mathrm{W}(5)$ & ${ }^{179 m} \mathrm{~W}$ & $5.2 \mathrm{~m}$ & W X-rays, 0.222 \\
\hline $\operatorname{Sm}(7)$ & ${ }^{153} \mathrm{Pm}$ & $5.5 \mathrm{~m}$ & $0.090 ? \cdot, 0.12,0.18$ \\
\hline $\mathrm{Hg}(1), \mathrm{Tl}(3), \mathrm{Pb}(4,9,10)$ & ${ }^{205} \mathrm{Hg}$ & $5.5 \mathrm{~m}$ & 0.205 \\
\hline $\operatorname{Ti}(1), V(3), \operatorname{Cr}(4,9,10)$ & ${ }^{51} \mathrm{Ti}$ & $5.80 \mathrm{~m}$ & $0.320(95 \%), 0.928(5 \%), \quad 0.605(1.5 \%)$ \\
\hline $\mathrm{Fe}(9,10)$ & ${ }^{56} \mathrm{Cr}$ & $5.9 \mathrm{~m}$ & $\begin{array}{l}{[\mathrm{Mn} \mathrm{X} \text {-rays }], 0.026,0.083} \\
\text { daughter radiations from }{ }^{56} \mathrm{Mn}\end{array}$ \\
\hline $\mathrm{Ni}(4,10)$ & ${ }^{61} \mathrm{Fe}$ & $6.0 \mathrm{~m}$ & $\begin{array}{l}\text { 1. } 20\left({ }^{\dagger} 100\right), 1.03(\dagger 98), 0.30\left({ }^{\dagger} 48\right), 0.13(\dagger 11), \cdots \\
\text { daughter radiations from }{ }^{61} \mathrm{Co}\end{array}$ \\
\hline $\operatorname{Kr}(3,8), \operatorname{Rb}(4,10)$ & ${ }^{84} \mathrm{Br}$ & $6.0 \mathrm{~m}$ & $1.46(75 \%), 0.88(75 \%), 0.44(68 \%), 1.89(16 \%)$ \\
\hline $\operatorname{Br}(1), \operatorname{Kr}(3,7,8), \operatorname{Rb}(4)$ & ${ }^{82 m} \mathrm{Br}$ & $6.1 \mathrm{~m}$ & $\begin{array}{l}\text { Br X-rays, } 0.046(0.3 \%), 0.777(0.15 \%) \text {, } \\
\quad 1.475(0.009 \%)\end{array}$ \\
\hline $\mathrm{Nb}(1), \operatorname{Mo}(3,7,8)$ & ${ }^{94 m} \mathrm{Nb}$ & $6.3 \mathrm{~m}$ & $\mathrm{Nb} \mathrm{X}$-rays, $0.871(0.2 \%), \cdots$ \\
\hline $\operatorname{Br}(5), \operatorname{Kr}(3,8)$ & ${ }^{78} \mathrm{Br}$ & $6.5 \mathrm{~m}$ & $0.511(184 \%)$, Se X-rays, $0.614(14 \%)$ \\
\hline $\operatorname{Sm}(3,8), \operatorname{Eu}(10)$ & ${ }^{152} \mathrm{Pm}$ & $6.5 \mathrm{~m}$ & {$[$ Sm X-rays $], 0.122,0.245$} \\
\hline $\operatorname{Pt}(4,10)$ & ${ }^{195} \mathrm{Os}$ & $6.5 \mathrm{~m}$ & $?$ \\
\hline $\mathrm{Si}(3,7), P(9)$ & ${ }^{29} \mathrm{Al}$ & $6.6 \mathrm{~m}$ & $1.28(94 \%), 2.43(6 \%)$ \\
\hline $\mathrm{Ce}(8)$ & ${ }^{134} \mathrm{La}$ & $6.7 \mathrm{~m}$ & $0.511(124 \%)$, Ba X-rays, $0.605(6 \%), \cdots$ \\
\hline $\mathrm{Te}(3)$ & ${ }^{130} \mathrm{Sb}$ & $7 \mathrm{~m}$ & $0.20,0.82$ (complex), $1.03,1.16$ \\
\hline $\operatorname{Pt}(7)$ & ${ }^{197} \mathrm{Ir}$ & $7 \mathrm{~m}$ & 0.50 \\
\hline $\operatorname{Dy}(3,7,8), \operatorname{Ho}(4)$ & ${ }^{162} \mathrm{~Tb}$ & $7.5 \mathrm{~m}$ & 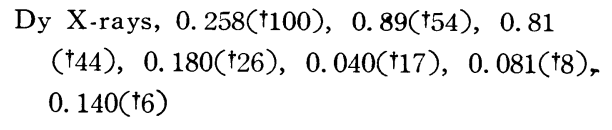 \\
\hline $\mathrm{K}(5), \mathrm{Ca}(8)$ & ${ }^{38} \mathrm{~K}$ & $7.71 \mathrm{~m}$ & $0.511(200 \%), 2.170(100 \%), \cdots$ \\
\hline $\mathrm{Ge}(3,8), \operatorname{As}(10)$ & ${ }^{74} \mathrm{Ga}$ & $7.9 \mathrm{~m}$ & $\begin{array}{l}0.60(100 \% \text {, doublet }), 2.35(45 \%), 0.50(11 \\
\% \text {, complex ? }), 0.87(9 \% \text {, doublet }) \\
1.46(8 \% \text {, doublet }), 1.20(8 \% \text {, doublet }) \\
1.76(7 \% \text {, doublet }), 1.33(5 \%), 1.11(5 \%)\end{array}$ \\
\hline $\operatorname{Zr}(4,10)$ & ${ }^{93} \mathrm{Sr}$ & $8 \mathrm{~m}$ & $\begin{array}{l}0.60\left({ }^{\dagger} 100\right), 0.8(\dagger 75), 1.2\left({ }^{\dagger} 18\right), 1.7\left({ }^{\dagger} 10\right) \\
0.3\left({ }^{\dagger}\right), \cdots \\
\text { daughter radiations from }{ }^{93} \mathrm{Y}\end{array}$ \\
\hline $\mathrm{Pb}(9,10)$ & ${ }^{206} \mathrm{Hg}$ & $8 \mathrm{~m}$ & $\begin{array}{l}0.31 \\
\text { daughter radiations from }{ }^{206} \mathrm{Tl}\end{array}$ \\
\hline $\mathrm{Fe}(5)$ & ${ }^{53} \mathrm{Fe}$ & $8.5 \mathrm{~m}$ & $0.511(196 \%), 0.38(32 \%)$ \\
\hline $\mathrm{Ca}(1), \operatorname{Ti}(10)$ & ${ }^{49} \mathrm{Ca}$ & $8.8 \mathrm{~m}$ & $\begin{array}{l}3.10(89 \%), 4.1(10 \%) \\
\text { daughter radiations from }{ }^{49} \mathrm{Sc}\end{array}$ \\
\hline $\operatorname{Sm}(5)$ & ${ }^{143} \mathrm{Sm}$ & $8.9 \mathrm{~m}$ & $0.511(100 \%)$, Pm X-rays \\
\hline $\mathrm{Se}(7), \operatorname{Br}(9)$ & ${ }^{79} \mathrm{As}$ & $\begin{array}{l}9.0 \mathrm{~m} \\
(68)\end{array}$ & $0.43(2 \%), \quad 0.36(2 \%), \quad 0.89(1 \%), 0.73(0.5$ \\
\hline
\end{tabular}




\begin{tabular}{|c|c|c|c|}
\hline $\begin{array}{l}\text { Element and } \\
\text { type of reaction }\end{array}$ & $\begin{array}{l}\text { Nuclide } \\
\text { formed }\end{array}$ & $\begin{array}{l}\text { Half } \\
\text { life }\end{array}$ & $\begin{array}{l}\text { Energies }(\mathrm{MeV}) \text { and intensities of } \gamma-, \cdots \\
\mathrm{X}-\text { and annihilation radiations }\end{array}$ \\
\hline & & & $\begin{array}{l}\%), 0.54(0.5 \%) \\
\text { daughter radiations from }{ }^{79 m} \mathrm{Se}\end{array}$ \\
\hline $\mathrm{Te}(10)$ & ${ }^{129} \mathrm{Sn}$ & $9 \mathrm{~m}$ & $1.15, \cdots$ \\
\hline $\mathrm{Xe}(3,7,8), \mathrm{Cs}(4)$ & ${ }^{130 m} \mathrm{I}$ & $9.2 \mathrm{~m}$ & $r$ \\
\hline$W(8)$ & ${ }^{178} \mathrm{Ta}$ & $9.4 \mathrm{~m}$ & $\begin{array}{l}\text { Hf X-rays, } 0.093(\dagger 100), 1.35(\dagger 46), 1.10\left(\dagger^{\dagger} 1\right) \text {, } \\
\text { 0. } 511(\dagger 10), 1.45(\dagger 9, \text { complex }), 1.18\left(\dagger^{\dagger},\right. \\
\text { complex) }\end{array}$ \\
\hline $\operatorname{Mg}(1), \operatorname{Al}(3), \operatorname{Si}(4,9,10)$ & ${ }^{27} \mathrm{Mg}$ & $9.5 \mathrm{~m}$ & $0.84(70 \%), 1.013(30 \%), 0.18(0.7 \%)$ \\
\hline $\mathrm{Ce}(3,8)$ & ${ }^{136} \mathrm{La}$ & $9.5 \mathrm{~m}$ & $\mathrm{Ba}$ X-rays, $0.511(66 \%), 0.818(2.5 \%)$ \\
\hline $\mathrm{Sn}(1), \mathrm{Te}(4,10)$ & ${ }^{125 m} \mathrm{Sn}$ & $9.7 \mathrm{~m}$ & $0.325(97 \%), \quad 1.39(0.8 \%), \cdots$ \\
\hline $\mathrm{Cu}(5), \operatorname{Zn}(8)$ & ${ }^{62} \mathrm{Cu}$ & $9.8 \mathrm{~m}$ & $0.511(195 \%), 1.17(0.5 \%$, complex $), \cdots$ \\
\hline $\operatorname{Os}(7), \operatorname{Ir}(9)$ & $〔 191] \mathrm{Re}$ & $9.8 \mathrm{~m}$ & $?$ \\
\hline $\operatorname{Os}(1,2), \operatorname{Ir}(7), \operatorname{Pt}(6,9)$ & ${ }^{190 m} \mathrm{Os}$ & $9.9 \mathrm{~m}$ & $\begin{array}{l}\text { Os X-rays, } 0.616(99 \%), 0.502(98 \%) \\
0.361(94 \%), 0.187(70 \%)\end{array}$ \\
\hline$N(5)$ & ${ }^{13} \mathrm{~N}$ & $10.0 \mathrm{~m}$ & $0.511(200 \%)$ \\
\hline $\operatorname{Sn}(7)$ & ${ }^{111 m} \operatorname{In}$ & $\approx 10 \mathrm{~m}$ & 0.53 \\
\hline $\operatorname{Sn}(4,10)$ & ${ }^{119} \mathrm{Cd}$ & $10 \mathrm{~m}$ & daughter radiations from ${ }^{119 m} \mathrm{In},{ }^{119} \mathrm{In}$ \\
\hline$W(3), \operatorname{Re}(10)$ & ${ }^{186} \mathrm{Ta}$ & $10 \mathrm{~m}$ & 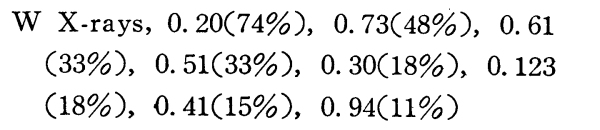 \\
\hline
\end{tabular}

